\title{
Importância dos padrões temporais no recurso ao atendimento complementar dos Centros de Saúde do Barreiro e Quinta da Lomba: pistas importantes para previsão da afluência
}

Frederico Rosário*

\section{RESUMO}

Objectivo: Analisar a relação entre factores temporais e o recurso ao Atendimento Complementar dos Centros de Saúde do Barreiro e Quinta da Lomba.

Tipo de estudo: Estudo observacional, transversal, analítico.

Local: Realizado nos Centros de Saúde do Barreiro e Quinta da Lomba.

População: Utentes que recorreram ao Atendimento Complementar durante o ano de 2007.

Métodos: Foi registado o número de inscrições no Atendimento Complementar em cada dia de 2007. Os dias foram caracterizados quanto a: mês do ano, dia da semana, se esse dia coincidia com um feriado, se tinha sido feriado no dia anterior, se tinha sido feriado no dia seguinte e se coincidia com tolerância de ponto. Determinou-se a importância das variáveis estudadas através do modelo de regressão de Poisson.

Resultados: Foi encontrada uma relação significativa entre todas as variáveis estudadas e o número de inscrições no Atendimento Complementar. À segunda-feira foram observados mais utentes do que nos restantes dias da semana, sendo o domingo o dia de menor afluência. Quando o dia da semana coincidiu com um feriado verificou-se que o número de inscrições nesse dia foi semelhante aos registados a um domingo. Nos dias a seguir aos feriados constatou-se uma tendência para maior afluência que o habitual, especialmente quando houve possibilidade de fim-de-semana prolongado. Observou-se o fenómeno oposto nos dias que precederam os feriados.

Conclusões: O recurso ao Atendimento Complementar é influenciado por factores temporais. O conhecimento destes padrões pode ser útil na previsão do número de doentes que recorre ao Atendimento Complementar, permitindo uma maior racionalização na distribuição dos recursos.

Palavras-Chave: Atendimento Complementar; Cuidados de Saúde Primários; Predição; Análise de Regressão.

\section{INTRODUÇÃO}

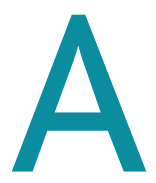

gestão dos cuidados de saúde está direccionada para os utilizadores, e a satisfação do utente é de primordial importância, expressa pelo valor que atribui aos cuidados presta- dos. ${ }^{1}$ Os serviços de saúde devem, portanto, procurar adequar a oferta de cuidados de saúde às necessidades da população por eles abrangida. A esta oferta de cui-

*Assistente Eventual de Medicina Geral e Familiar, Centro de Saúde Quinta da Lomba. 
dados não é alheia a prestação de cuidados de urgência.

O uso dos serviços de urgência é uma das fontes de preocupação nos países desenvolvidos, ${ }^{2,3}$ e um tema de pesquisa recorrente na literatura. ${ }^{4}$ Com o intuito de descongestionar os serviços de urgência hospitalar foram criados nos Centros de Saúde os Serviços de Atendimento Permanente e os Serviços de Atendimento Complementar. ${ }^{5}$ Os dados disponíveis para Portugal estimavam um total de 8,5 milhões de consultas urgentes anuais em 1996, 40\% delas realizadas a nível dos Cuidados de Saúde Primários, ${ }^{1}$ valor esse que aumentou para 11,6 milhões em $2004 .^{6}$

O Atendimento Complementar dos Centros de Saúde do Barreiro e Quinta da Lomba serve uma população de cerca de 80 mil habitantes, com o Hospital de referência situado sensivelmente a igual distância entre os dois Serviços de Atendimento Complementar (cerca de 2 quilómetros). Durante o ano de 2007, o Atendimento Complementar funcionou por um período de 12 horas, entre as 10:00 e as 22:00.

Têm sido muitas vezes referidas, em conversas informais, associações entre factores temporais e o recurso ao Atendimento Complementar nestes Centros de Saúde. É exemplo a noção empírica que a afluência é maior às segundas-feiras. Contudo, não se encontram na literatura estudos que demonstrem a existência destas associações. O seu conhecimento poderia ser um aspecto interessante a ter em conta na melhoria da resposta às necessidades dos utentes. O objectivo deste estudo foi o de analisar a relação entre factores temporais e o recurso ao Atendimento Complementar dos Centros de Saúde do Barreiro e Quinta da Lomba.

\section{MÉTODOS}

Estudo observacional, transversal, analítico, realizado no Atendimento Complementar dos Centros de Saúde do Barreiro e Quinta da Lomba. Com base no programa Sinus ${ }^{\circledR}$ foi registado o número de inscrições no Atendimento Complementar em cada dia do ano de 2007. Cada dia do ano foi ainda caracterizado quanto a: mês do ano, dia da semana, se esse dia coincidia com um feriado, se tinha sido feriado no dia anterior, se tinha sido feriado no dia seguinte e se esse dia coincidia com tolerância de ponto. Foram excluídos da análise os dias correspondentes a greve da função pública: dia 30 de Maio e dia 30 de Novembro.
A importância das variáveis estudadas foi determinada através do modelo de regressão de Poisson para contagens com ligação identidade. Consideraram-se significativas as variáveis que, descontando o efeito das restantes variáveis no modelo, apresentaram um valor $p$ menor ou igual a 0,05 . Sempre que os graus de liberdade do modelo o permitiram, foram analisadas as interacções entre as variáveis explicativas. Determinou-se que o domingo do mês de Agosto que não era antecedido, precedido ou coincidente com um feriado, seria usado como termo de comparação. O número de inscrições esperadas por hora foi determinado dividindo o número total de inscrições esperadas pelo número de horas de funcionamento do Atendimento Complementar. A importância de cada uma das variáveis estudadas foi avaliada pela magnitude de redução da deviance face ao modelo nulo, ou seja, pela redução na variabilidade causada pela introdução das variáveis no modelo. $\mathrm{O}$ tratamento dos dados foi realizado recorrendo aos softwares estatísticos $\mathrm{R}^{\circledR} 2.7 .0$ (The R Foundation for Statistical Computing), SPSS ${ }^{\circledR} 13.0$ (SPSS for Windows, Rel. 13.0. 2004. Chicago: SPSS Inc.) e Microsoft $^{\circledR}$ Excel 2002.

\section{RESULTADOS}

\section{Análise Descritiva}

Foi registada a afluência ao Atendimento Complementar dos Centros de Saúde do Barreiro e Quinta da Lomba em 363 dias do ano de 2007. O número de inscrições registadas variou entre um mínimo de 61 no dia 29 de Julho e um máximo de 333 a 4 de Janeiro. No total foram contabilizadas 30919 consultas, $5,5 \%$ das quais necessitaram de referenciação hospitalar. A análise do número de inscrições por dia da semana (Figura 1) mostrou uma clara tendência para um maior recurso ao Atendimento Complementar à segunda-feira do que nos restantes dias da semana $(198,4 \pm 5,8)$. O domingo foi o dia de menor afluência $(105,0 \pm 3,4)$. A mesma análise para a afluência por mês do ano (Figura 2) revelou que Fevereiro foi o mês de maior afluência média (222,0 \pm 9 ,3) enquanto em Agosto se observou um menor número de inscrições no Atendimento Complementar $(121,5 \pm 5,9)$.

Modelo de Regressão de Poisson

A análise através da regressão de Poisson foi usada para 


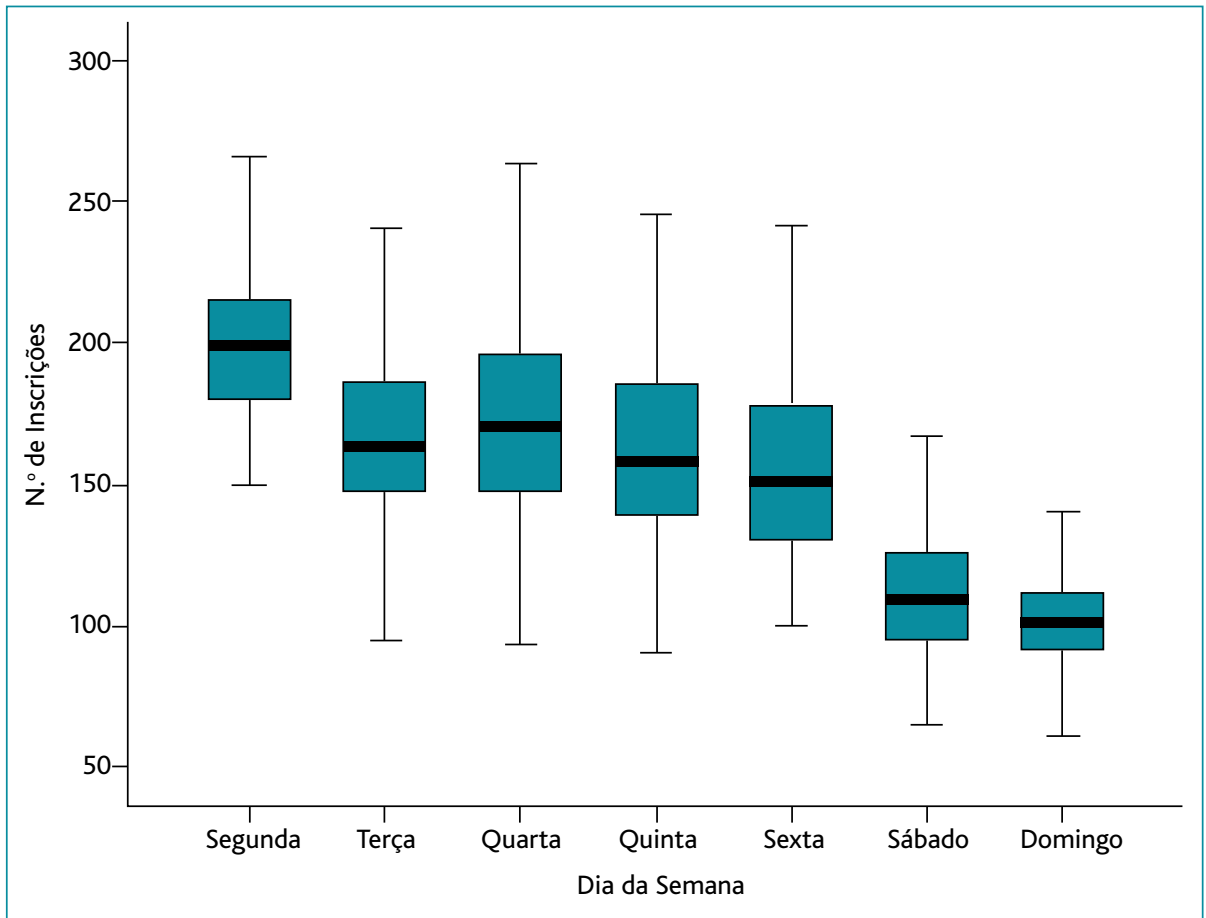

Figura 1. Distribuição do número de inscrições no Atendimento Complementar por dia da semana. o mês de Agosto, verifica-se que em Julho e Setembro foram observados em média mais cerca de 6 utentes por dia $(p=0,001$ e $p=0,006$, respectivamente), ao passo que em Fevereiro foram registadas mais cerca de 100 idas ao Atendimento Complementar, ou seja, aproximadamente mais 8 utentes por hora (Quadro II).

A análise da influência do feriado no recurso ao Atendimento Complementar dos Centros de Saúde do Barreiro e Quinta da Lomba mostrou que não se observaram alterações significativas quando este coincide com um domingo (aproximadamente menos 6 inscrições do que a um domingo em que não é feriado, o determinar quais as variáveis que influenciavam significativamente o recurso ao Atendimento Complementar nos Centros de Saúde do Barreiro e Quinta da Lomba, descontando o efeito das restantes variáveis. A dimensão da amostra permitiu analisar a influência de dois tipos de interacções: 1) interacção entre o dia da semana e o feriado, ou seja, em que medida é que ser feriado altera o recurso ao Atendimento Complementar; 2) interacção entre o dia da semana e ter sido feriado no dia anterior, ou seja, que influência tem, no número de inscrições, o facto de o dia anterior ter sido feriado. Todas as variáveis e interacções introduzidas no modelo foram significativas (Quadro I). O modelo resultante pode ser consultado em detalhe no Quadro II.

Com base no modelo obtido, a afluência ao Atendimento Complementar nestes Centros de Saúde foi significativamente maior em qualquer dia da semana quando comparado com o domingo, sendo que a maior diferença é registada à segunda-feira, em que foram registadas mais cerca de 94 inscrições diárias $(p<0,001)$, o que corresponde a um acréscimo de aproximadamente 8 utentes por hora (Quadro II).

Analisando os meses do ano, e em comparação com que corresponde a menos cerca de um doente a cada duas horas, $p=0,358$ ). A menor afluência verificou-se quando o feriado coincide com uma segunda-feira, aproximadamente menos 128 inscrições do que a uma segunda-feira regular $(p<0,001)$, ou seja, sensivelmente menos 11 idas por hora ao Atendimento Complementar. Ao contrário dos restantes dias, observou-se um aumento significativo da afluência quando o feriado coincide com um sábado (Quadro II).

A ocorrência de um feriado no dia anterior ao domingo não promoveu alterações significativas na afluência nesse dia (menos cerca de uma inscrição a cada duas horas, $p=0,389$ ). Por outras palavras, quando um feriado coincide com um sábado, o número de inscrições no domingo seguinte não parece diferir de um outro domingo qualquer. Segundo o modelo obtido, a presença de um feriado no dia anterior aumentou de forma significativa o número de inscrições às sextas, terças e quartas-feiras, sendo mais marcado o aumento nesta última (mais cerca de 5 utentes por hora, $p<$ 0,001) (Quadro II).

A tolerância de ponto reduziu o número de inscrições (Quadro II). 
O modelo explicou 85,9\% da variabilidade no recurso ao Atendimento Complementar. De todas as variáveis estudadas o dia da semana foi a variável que produziu a maior explicação da variabilidade (45,8\%) (Quadro I).

\section{DISCUSSÃO}

Os resultados deste estudo mostram a existência de padrões temporais no recurso ao Atendimento Complementar dos Centros de Saúde do Barreiro e Quinta da Lomba, uma vez que estes factores parecem ter influência no número de inscrições neste serviço, o que vem corroborar a ideia da importância dos motivos sociais $^{7,8}$ no recurso ao Atendimento Complementar.
Dos padrões temporais encontrados, o dia da semana foi o que mostrou ter maior importância, tendo-se verificado uma tendência para um maior número de inscrições à segunda-feira. Neste dia foram observados em média mais cerca de 94 utentes do que a um Domingo, o dia da semana com menor afluência, dando razão à noção empírica de ser a segunda-feira o dia de maior movimento no Atendimento Complementar dos Centros de Saúde do Barreiro e Quinta da Lomba. A importância desta diferença é realçada quando se avalia a quantidade de trabalho, traduzida numa média de mais cerca de 8 utentes por hora à segunda-feira. A inexistência de estudos semelhantes não permite comparar estes valores nem tão pouco verificar se este é um

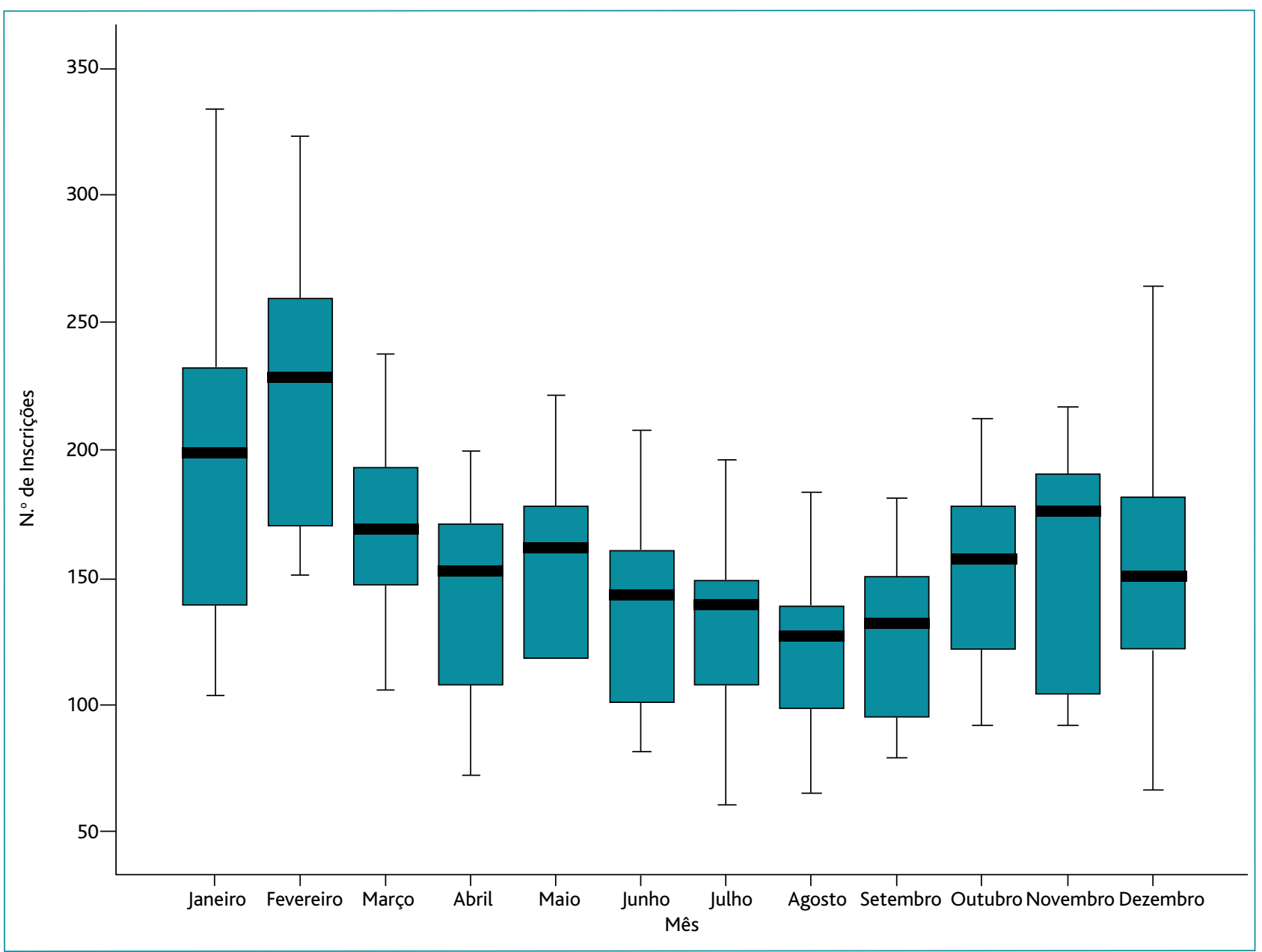

Figura 2. Distribuição do número de inscrições no Atendimento Complementar por mês do ano. 


\begin{tabular}{|c|c|c|c|c|c|}
\hline & $\begin{array}{l}\text { Graus de } \\
\text { Liberdade }\end{array}$ & Deviance & $\begin{array}{l}\text { Deviance } \\
\text { Residual }\end{array}$ & valor $p$ & $\begin{array}{l}\text { Variabilidade } \\
\text { explicada (\%) }\end{array}$ \\
\hline Modelo Nulo & 362 & -- & 5294.6 & -- & 0 \\
\hline Dia da Semana & 6 & 2423.9 & 2870.8 & $<0,001$ & 45,8 \\
\hline Mês & 11 & 1567.4 & 1303.3 & $<0,001$ & 29,6 \\
\hline Feriado & 1 & 186.5 & 1116.8 & $<0,001$ & 3,5 \\
\hline Feriado Dia Anterior & 1 & 16.1 & 1100.7 & $<0,001$ & 0,3 \\
\hline Feriado Dia Seguinte & 1 & 46.4 & 1054.3 & $<0,001$ & 0,9 \\
\hline Tolerância & 1 & 42.3 & 1012.1 & $<0,001$ & 0,8 \\
\hline Dia * Feriado & 6 & 230.7 & 781.4 & $<0,001$ & 4,4 \\
\hline Dia * Fer. Dia Ant. & 6 & 32.2 & 749.1 & $<0,001$ & 0,6 \\
\hline
\end{tabular}

Dia * Feriado - Interacção do Dia da Semana com o Feriado; Dia * Fer. Dia Ant. - Interacção do Dia da Semana com o Feriado no Dia Anterior

padrão comum a outros locais do país ou um acontecimento local específico do Barreiro. Ficam por esclarecer quais os motivos de tal fenómeno. Tem-se argumentado, ainda que sem ter sido demonstrado, que a maior afluência neste dia está relacionada com o desejo dos utentes em conseguir justificação médica para não comparecer nos seus postos de trabalho após o fim-de-semana. Esta hipótese estaria de acordo com outros estudos que realçam a importância de factores socioculturais na decisão de recorrer a serviços de urgência. ${ }^{7,8}$

Outro aspecto interessante deste estudo relaciona-se com a influência dos feriados no número de inscrições. Como seria de esperar, ser feriado a um domingo não alterou de forma significativa o número de utentes que recorre a este serviço. Contudo, com a notável excepção do sábado, verificou-se que o feriado condicionou reduções significativas no número de inscrições. Salienta-se que a redução esperada para as quartas, quintas e sextas-feiras é semelhante ao aumento de afluência nestes dias quando não é feriado, ou seja, quando um feriado coincide com um destes dias da semana, eles comportam-se como se de um domingo se tratasse. $\mathrm{Ou}$ tro fenómeno a salientar é que a magnitude desta redução na afluência vai-se esbatendo de segunda para sexta-feira, verificando-se uma redução desproporcionada às segunda e terça feiras quando estes dias coincidem com um feriado. Nestes dias são esperadas menos cerca de 11 e 7 inscrições por hora, respectivamente, reduzindo em muito a quantidade de trabalho. As razões para tais achados parecem difíceis de compreender. Poder-se-á argumentar que estes dias são usados como fim-de-semana prolongado. Contudo, o mesmo argumento deveria ser válido para quinta e sexta feiras e nestes dias a redução não é tão acentuada. Ficam ainda por explicar as razões que levam a um aumento do número de idas à urgência nos feriados ao sábado.

Verificou-se ainda que o feriado influenciou o recurso ao Atendimento Complementar dos Centros de Saúde do Barreiro e Quinta da Lomba tanto no dia "pós-feriado» como no dia que o precede. Assim, um feriado à quarta-feira, sexta-feira, sábado ou domingo não aumentou de forma significativa o número de utentes que se deslocou ao Atendimento Complementar nos dias imediatamente a seguir, ou seja, quinta-feira, sábado, domingo e segundafeira, respectivamente. Para os restantes dias, a presença de um feriado no dia anterior condicionou um aumento na afluência. Verificou-se ainda que este aumento foi maior à quarta-feira, ou seja, um feriado a uma terça-feira aumentou a afluência na quarta-feira seguinte. Este acréscimo é quantificado em mais cerca de 5 utentes por hora. Mais uma vez, a possibilidade dos utentes gozarem um fim-de-semana prolongado parece alterar os seus padrões de utilização do Atendimento Complementar nestes Centros de Saúde.

Verificou-se o fenómeno contrário no dia que precede o feriado, ou seja, foram observados menos utentes nestes dias. A dimensão da amostra não permitiu uma análise individualizada deste efeito para cada dia da semana, não se podendo verificar especificamente quais os dias em que este efeito é significativo.

Nos dias em que foi concedida tolerância de ponto, de forma similar aos dias de feriado, verificou-se uma redução acentuada no número de inscrições. Também aqui não foi possível individualizar o efeito que tem este acontecimento em cada dia da semana.

A competência cultural, ou seja, o conhecimento dos 


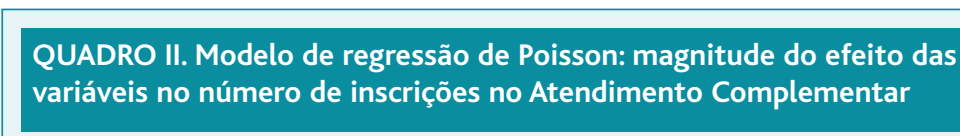

\begin{tabular}{|l|l|l|l|l}
\hline Referência & 73,7 & 2,3 & $<0,001$ & 6,1 \\
\hline Sábado & 7,0 & 2,1 & $<0,001$ & 0,6 \\
\hline Sexta & 51,7 & 2,3 & $<0,001$ & 4,3 \\
\hline Quinta & 62,1 & 2,4 & $<0,001$ & 5,2 \\
\hline Terça & 64,8 & 2,4 & $<0,001$ & 5,4 \\
\hline Quarta & 69,3 & 2,4 & $<0,001$ & 5,8 \\
\hline Segunda & 94,2 & 2,5 & $<0,001$ & 7,9 \\
\hline Setembro & 7,7 & 2,8 & 0,006 & 0,6 \\
\hline Julho & 8,9 & 2,8 & 0,001 & 0,7 \\
\hline
\end{tabular}

\begin{tabular}{|l|l|l|l|l}
\hline Junho & 8,9 & 2,8 & 0,001 & 0,7 \\
\hline Abril & 20,1 & 3,0 & $<0,001$ & 1,7 \\
\hline Outubro & 24,3 & 2,9 & $<0,001$ & 2,0 \\
\hline
\end{tabular}

\begin{tabular}{|c|c|c|c|c|}
\hline & & & & \\
\hline Maio & 30,6 & 3,0 & $<0,001$ & 2,5 \\
\hline Novembro & 34,6 & 3,0 & $<0,001$ & 2,9 \\
\hline Dezembro & 37,5 & 3,1 & $<0,001$ & 3,1 \\
\hline Março & 46,5 & 3,0 & $<0,001$ & 3,9 \\
\hline Janeiro & 69,2 & 3,2 & $<0,001$ & 5,8 \\
\hline Fevereiro & 100,1 & 3,4 & $<0,001$ & 8,3 \\
\hline F. Segunda & $-127,6$ & 12,8 & $<0,001$ & $-10,6$ \\
\hline F. Terça & $-85,3$ & 9,8 & $<0,001$ & $-7,1$ \\
\hline F. Quarta & $-66,6$ & 9,7 & $<0,001$ & $-5,5$ \\
\hline F. Quinta & $-56,5$ & 9,2 & $<0,001$ & $-4,7$ \\
\hline F. Sexta & $-43,6$ & 10,2 & $<0,001$ & $-3,6$ \\
\hline F. Domingo & $-6,4$ & 7,0 & 0,358 & $-0,5$ \\
\hline F. Sábado & 24,7 & 11,2 & 0,028 & 2,1 \\
\hline Fda. Domingo & $-6,7$ & 7,8 & 0,389 & $-0,6$ \\
\hline Fda. Sábado & 3,1 & 10,8 & 0,775 & 0,3 \\
\hline Fda. Quinta & 15,6 & 12,0 & 0,192 & 1,3 \\
\hline Fda. Segunda & 16,2 & 12,9 & 0,211 & 1,3 \\
\hline Fda. Sexta & 39,4 & 11,3 & $<0,001$ & 3,3 \\
\hline Fda. Terça & 46,1 & 17,8 & 0,009 & 3,8 \\
\hline Fda. Quarta & 56,1 & 13,2 & $<0,001$ & 4,7 \\
\hline Fds. & $-13,2$ & 3,7 & $<0,001$ & $-1,1$ \\
\hline Tolerância & $-45,3$ & 6,3 & $<0,001$ & $-3,8$ \\
\hline
\end{tabular}

Referência - Domingo, mês de Agosto, não feriado, não tolerância, sem feriado no anterior nem no dia seguinte; F. - Feriado; Fda. - Feriado no dia anterior; Fds. - Feriado no dia seguinte

\begin{tabular}{l|l|l|l|l} 
Factor & $\begin{array}{l}\text { N. }{ }^{\circ} \text { Inscrições } \\
\text { Esperadas }\end{array}$ & $\begin{array}{l}\text { Desvio- } \\
\text {-Padrão }\end{array}$ & valor $\mathrm{p}$ & $\begin{array}{l}\text { Acréscimo de } \\
\text { Inscrições por } \\
\text { hora (média) }\end{array}$ \\
\hline Referência & 73,7 & 2,3 & $<0,001$ & 6,1 \\
\hline Sábado & 7,0 & 2,1 & $<0,001$ & 0,6
\end{tabular}

Atendimento Complementar pode ser usado para elaboração de modelos de predição que facilitem a distribuição de recursos materiais e humanos, com inegáveis ganhos económicos e na qualidade do serviço prestado. O modelo proposto para os Centros de Saúde do Barreiro e Quinta da Lomba explicou $85,9 \%$ da variabilidade no recurso ao Atendimento Complementar. Este valor deixa antever a potencial utilidade do conhecimento destes padrões na elaboração de modelos preditivos da afluência ao Atendimento Complementar. A previsão do número de utentes que recorre a este serviço poderá permitir a flexibilização das escalas de serviço dos profissionais do Centro de Saúde, ajustando o seu número às necessidades da população. Este ajuste poderá ser importante na melhoria, não só dos cuidados de saúde prestados, mas também no grau de satisfação de utentes e profissionais.

Podem ser apontados como pontos fortes deste trabalho, por um lado a abordagem inovadora ao problema da elevada afluência ao Atendimento Complementar, quer nas variáveis estudadas, quer na metodologia utilizada, o que o torna pioneiro nesta área de investigação; por outro lado o facto de deixar antever a sua aplicabilidade na optimização dos recursos com o intuito de melhorar os cuidados prestados à população.

Devem ser tidas em conta algumas limitações deste estudo. O modelo apresentado, ainda que promissor, não pode ser aplicado nos Centros de Saúde onde decorreu o estudo uma vez que não foi efectuada a sua validação interna. Por outro lado deve ter-se presente que dificilmente este modelo poderá ser extrapolado para outros Centros de Saúde com populações e horários de funcionamento diferentes, o que não invalida que se contextos sociais e culturais da população utente dos serviços de saúde, é um aspecto importante dos cuidados de saúde, assumindo particular importância ao nível dos cuidados de saúde primários. ${ }^{9}$ Neste caso particular, o reconhecimento de padrões no recurso ao possa utilizar a mesma metodologia no desenho de um modelo adaptado a cada Centro de Saúde. Por fim é de esperar que o presente modelo não se adeqúe a períodos de epidemia, situação em que será mais recomendável o uso de modelos epidémicos. 


\section{REFERÊNCIAS BIBLIOGRÁFICAS}

1. Oliveira A. Urgência básica. Acta Med Port 2006 Jul-Aug; 19 (4): 269-73 .

2. Pereira S, Oliveira e Silva A, Quintas M, Almeida J, Marujo C, Pizarro M, et al. Appropriateness of emergency department visits in a Portuguese university hospital. Ann Emerg Med 2001 Jun; 37 (6): 580-6.

3. Northington WE, Brice JH, Zou B. Use of an emergency department by nonurgent patients. Am J Emerg Med 2005 Mar;23 (2): 131-7.

4. Harris L, Bombin $M, C h i F$, DeBortoli $T$, Long J. Use of the emergency room in Elliot Lake, a rural community of Northern Ontario, Canada. Rural Remote Health 2004 Jan-Mar; 4 (1): 240.

5. Palma J. Problemas de saúde urgentes: a quem recorrem os utentes e o que influencia a sua escolha. Rev Port Clin Geral 2002 Set-Out; 18 (5): 283-97.

6. Oliveira A. Requalificação da rede da urgência/emergência: mudança certa ou errada? Rev Port Med Interna 2008 Out-Dez;15 (4): 237-44.
7. Padgett DK, Brodsky B. Psychosocial factors influencing non-urgent use of the emergency room: a review of the literature and recommendations for research and improved service delivery. Soc Sci Med 1992 Nov; 35 (9): 1189-97.

8. Petersen LA, Burstin HR, O'Neil AC, Orav EJ, Brennan TA. Nonurgent emergency department visits: the effect of having a regular doctor. Med Care 1998 Aug; 36 (8): 1249-55.

9. South-Paul JE, Lewis EL, Matheny SC. Current Diagnosis \& Treatment in Family Medicine. New York, NY: McGraw-Hill; 2004.

\section{ENDEREÇO PARA CORRESPONDÊNCIA}

Frederico Rosário

Centro de Saúde do Barreiro, Rua D. José Cárcamo Lobo

2835-423 Lavradio

Recebido em 26/03/2009

Aceite para publicação em 22/05/2010

\section{ABSTRACT}

\section{TEMPORAL PATTERNS IMPORTANCE IN AFTER-HOURS CARE VISITS AT THE BARREIRO AND QUINTA DA LOMBA PRIMARY CARE CENTERS: IMPORTANT CLUES TO PREDICTION}

Objectives: To analyze the association between temporal factors and after-hours care of the Barreiro and Quinta da Lomba Primary Care Centers.

Design: Observational, cross-sectional, analytic study

Setting: Performed at the Barreiro and Quinta da Lomba Primary Care Centers.

Population: Patients that used the after-hours care during 2007.

Methods: The number of emergency department visits during 2007 was considered the outcome variable while the month, day of the week, holydays, presence of a holyday in the previous day, presence of a holyday in the following day and work tolerance were considered as independent variables. Independent and outcome variables associations were determined with the Poisson regression model.

Results: A significant association was found between the outcome variable and all the independent variables. More visits are expected at Monday than in any other day being Sunday the day with the lowest number of visits. Holydays reduced the number of after-hours consultation, making it similar to Sundays. In the days that followed a holyday there was a tendency for more after-hours visits, especially if there was a prolonged weekend possibility. The opposite was true in those days that preceded these events.

Conclusions: After-hours care is influenced by temporal factors. This knowledge can be useful in forecasting the number of after-hours consultations, allowing a more rational distribution of resources.

Keywords: After-Hours Care; Primary Health Care; Forecasting; Regression Analysis. 